\title{
Dynamics of Flux Creep in Underdoped Single Crystals of $\mathrm{Y}_{1-x} \mathrm{Pr}_{x} \mathrm{Ba}_{2} \mathrm{Cu}_{3} \mathrm{O}_{7-\delta}$.
}

\author{
T. Stein, G. A. Levin, and C. C. Almasan \\ Department of Physics, Kent State University, Kent OH 44242 \\ D. A. Gajewski and M. B. Maple \\ Department of Physics and Institute for Pure and Applied Physical Sciences, University of California, San Diego, La Jolla, \\ CA 92093
}

\begin{abstract}
Transport as well as magnetic relaxation properties of the mixed state were studied on strongly underdoped $\mathrm{Y}_{1-x} \mathrm{Pr}_{x} \mathrm{Ba}_{2} \mathrm{Cu}_{3} \mathrm{O}_{7-\delta}$ crystals. We observed two correlated phenomena - a coupling transition and a transition to quantum creep. The distribution of transport current below the coupling transition is highly nonuniform, which facilitates quantum creep. We speculate that in the mixed state below the coupling transition, where dissipation is nonohmic, the current distribution may be unstable with respect to self-channeling resulting in the formation of very thin current-carrying layers.
\end{abstract}

\section{INTRODUCTION}

The majority of experiments devoted to the study of dissipation in the mixed state of cuprate superconductors were performed on optimally doped (maximum critical temperature $T_{c}$ ) single crystals, thin films, or superlatttices. Meanwhile, in underdoped systems, the normal state properties exhibit a number of unusual features which quite possibly hold a key to the understanding of the nature of the normal and superconducting states in cuprates. Also, as a result of the lower $T_{c}$ and upper critical field $H_{c 2}$, the dissipation in underdoped systems can be measured down to much lower reduced temperatures $T / T_{c}$ in relatively small fields of a few tesla. In contrast, in optimally doped single crystals, the dissipation falls below typically detectable levels at much higher reduced temperatures. Thus, underdoped cuprates facilitate the study of a broader range of the magnetic field - temperature $H-T$ phase diagram of the "vortex matter" than optimally doped superconductors. These facts provide a compelling reason to undertake a comprehensive study of the mixed state of strongly underdoped cuprates, including both transport and magnetic relaxation measurements.

One of the outstanding and extensively debated questions is the nature of the coupling transition in layered vortex systems. A sharp coupling transition has been observed in superconductor/insulator multilayers beginning with the pioneering work of Giaever [1], and later in Refs. [2, 3]. In a magnetic field $H$ applied normally to the planes, the positions of $2 \mathrm{D}$ vortices (pancakes) on neighboring superconducting layers tend to be uncorrelated at high temperatures. This vortex state is called $2 \mathrm{D}$ liquid. As the temperature decreases, the correlation in the direction of the applied magnetic field strengthens and the vortices tend to form coherent $3 \mathrm{D}$ flexible lines. For high- $T_{c}$ superconductors, though, the existence of the coupling transition is not as obvious. Several groups us- ing a 6-terminal (flux transformer) technique have arrived at conflicting conclusions.

Safar et al. [4] reported the observation of a transition to a $3 \mathrm{D}$ liquid in $\mathrm{YBa}_{2} \mathrm{Cu}_{3} \mathrm{O}_{7-\delta}$ which manifests itself as a convergence of voltages generated by the motion of vortices on opposite faces of the sample. However, such a strong manifestation of the coupling transition appears to be the exception rather than the rule. Other groups have observed that voltages generated on opposite faces of $\mathrm{Bi}_{2} \mathrm{Sr}_{2} \mathrm{CaCu}_{2} \mathrm{O}_{8}$ [5, 8] and $\mathrm{YBa}_{2} \mathrm{Cu}_{3} \mathrm{O}_{7-\delta}$ [9] single crystals diverge with lowering temperature, rather than converge.

A point of view which can reconcile the results of these experiments is that a significant increase in the correlation length $L_{c}$ of the vortices in the c-direction indeed takes place at a well defined temperature, but $L_{c}$ may remain smaller than the thickness of the sample. Thus, voltages generated on opposite faces of the sample may never converge in spite of a macroscopic correlation length. The underdoped cuprates present an opportunity to test this idea for reasons presented above. If the coupling transition can be proven in an underdoped system, it must also occur in optimally doped cuprates since the coupling between the $\mathrm{CuO}$ bilayers decreases with underdoping.

A second important question in the physics of vortex matter is the possibility of non-activated, temperature independent, creep due to quantum rather than classical (over the barrier) relaxation at low temperatures. Superconductors represent, perhaps, the only system in which relaxation due to quantum creep is an experimentally accessible phenomenon. Here, a strongly non-equilibrium macroscopic metastable state relaxes coherently without thermal activation. In contrast, in the majority of other macroscopic metastable systems, relaxation proceeds as a sequence of a large number of uncorrelated microscopic steps, requiring thermal activation over an energy barrier.

Yet, the evidence of a magnetic relaxation rate that 
does not extrapolate to zero as $T \rightarrow 0$ has not reached a point where experimental data can form a cohesive picture of the phenomenon. While non-vanishing magnetic relaxation has been observed in both single crystals and thin films 10 13, non-vanishing resistance has been observed only in ultrathin films 14 16]. This has contributed to the assertion that temperature independent resistance in films and non-vanishing low-temperature magnetic relaxation in single crystals are unrelated phenomena. Since these two types of measurements involve different ranges of current (small currents in transport and large currents, close to the critical current $J_{c}$, in magnetic relaxation), they are open to alternative interpretations, not related to quantum creep. For example, Gerber and Franse [17 have argued that non-vanishing magnetic relaxation at low temperatures may result from self-heating, so that the local temperature of the sample is higher than that of the ambient.

The best way to address these issues is to conduct both types of measurements on the same system. A signature of a temperature independent creep appearing in both transport and magnetic relaxation measurements at the same temperature would be convincing proof that this phenomenon is not an artifact and reflects a fundamental change in the relaxation process.

The strongly underdoped $Y_{1-x} \mathrm{Pr}_{x} \mathrm{Ba}_{2} \mathrm{Cu}_{3} \mathrm{O}_{7-\delta}\left(T_{c} \approx\right.$ $17-21 K)$ system is an excellent candidate for this study. One interesting aspect of this low $T_{c}$ system is that its normal state, revealed by the suppression of superconductivity by magnetic field, is a two-dimensional insulator [18. The reduced dissipation in the normal core of a vortex (due to a large normal-state resistivity $\rho_{n}$ ) increases the mobility of the vortices 19 and, therefore, favors quantum tunneling 20 22]. Thus, in such an insulator-superconductor material we can expect that the transition from thermally activated to quantum creep takes place at higher temperatures than in conventional superconductors or in more metallic cuprates with higher $T_{c}$.

In addition, as shown below, this insulatorsuperconductor system provides an example of a drastic departure from the current theoretical understanding of the quantum creep phenomenon. Extrapolation of the theoretical results for dirty superconductors leads to the conclusion that the zero temperature magnetic relaxation rate scales with the zero temperature normal state conductivity $\sigma_{n}$. Contrary to this, as shown below, the magnetic relaxation rate of $\mathrm{Y}_{.47} \mathrm{Pr}_{.53} \mathrm{Ba}_{2} \mathrm{Cu}_{3} \mathrm{O}_{7-\delta}$ remains finite in spite of $\sigma_{n}(T) \rightarrow 0$ at $T \rightarrow 0$.

We reported the observation of quantum creep in $\mathrm{Y}_{1-x} \mathrm{Pr}_{x} \mathrm{Ba}_{2} \mathrm{Cu}_{3} \mathrm{O}_{7-\delta}$ crystals in a recent Letter [23. In this paper, we present more data and an extended analysis of both transport and magnetic relaxation measurements on two strongly underdoped single crystals of $Y_{.47} \mathrm{Pr}_{.53} \mathrm{Ba}_{2} \mathrm{Cu}_{3} \mathrm{O}_{7-\delta}$ with $\mathrm{T}_{c} \approx 17$ and $21 \mathrm{~K}$, respectively. The two twinned single crystals were grown by a self-flux technique as described elsewhere [24]. Typical dimensions are $0.8 \times 0.5 \times 0.015 \mathrm{~mm}^{3}$, with the c-axis of the crystals oriented along the smallest dimension.

By performing transport measurements as a function of applied current $I$ and magnetic field $H$, we demonstrate the existence of a current independent coupling transition temperature $T^{*}(H)$ preceding the crossover to quantum creep. The dissipation is ohmic above $T^{*}$ and non-ohmic below $T^{*}$. A picture that arises from these observations is that the sample, at $T<T^{*}$, is divided into two macroscopic regions: a layer near the primary face (where the current contacts are located) which carries most of the transport current, and the rest of the sample which remains mostly undisturbed by the current. Inside these layers the vortices are coupled, with the correlation length comparable to the thickness of the respective layer. On the other hand, these two macroscopic regions are decoupled from each other. As a result, the ratio $V_{p} / V_{s}$ of the primary to the secondary voltage increases with decreasing temperature several orders of magnitude over its value in the normal state.

The crossover to temperature independent (quantum) creep takes place at $T=T_{q}(H)<T^{*}(H)$ and only in the top (current-carrying) layer; the rest of the sample continues to exhibit thermally activated creep. This leads us to the conclusion that the upper layer has a thickness of only a few unit cells similar to ultrathin films and multilayers, the only other systems in which quantum creep has been observed in transport. Outside this region, the vortices have much greater length, which suppresses quantum tunneling because the probability of tunneling decreases exponentially with the length of the tunneling segment. We argue below that the formation of one or several very thin channels that carry a current density much greater than average may be a result of non-ohmic dissipation below $T^{*}$ which can lead to an instability of the current distribution with respect to self-channeling.

In order to confirm that the T-independent dissipation is due to quantum tunneling, we performed magnetic relaxation measurements on a similar crystal of $Y_{1-x} \mathrm{Pr}_{x} \mathrm{Ba}_{2} \mathrm{Cu}_{3} \mathrm{O}_{7-\delta}$. The results show a transition to $\mathrm{T}$-independent relaxation rate at approximately the same temperature $T_{q}(H)$ as in transport. In addition, we were able to determine the characteristic relaxation time which characterizes the rate of relaxation uninhibited by the lack of thermal energy. The value of this "escape time" $1 s$ indicates that the relaxation of the magnetic moment is governed by the diffusion of vortices from the bulk to the outer edge of the sample.

\section{TRANSPORT MEASUREMENTS}

Transport measurements were performed using the "flux transformer" contact configuration (Inset in Fig. 11). The current $I$ was injected through the contacts on one 


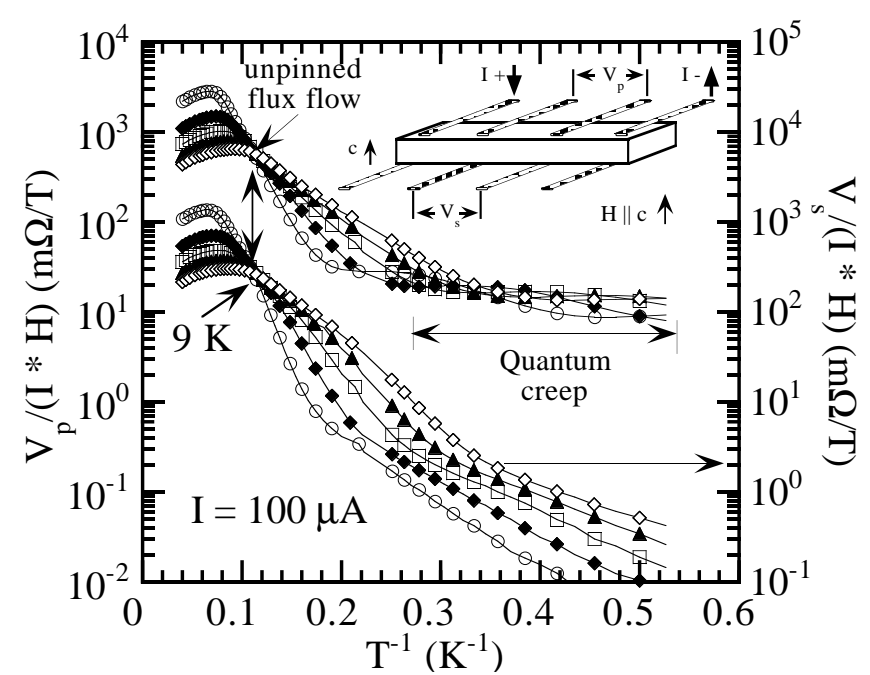

FIG. 1. Primary $V_{p}$ and secondary $V_{s}$ voltages normalized to the total current $I$ and magnetic field $H$ plotted versus $1 / T$ for five magnetic fields $(0.2,0.4,0.60 .8$, and $1 T)$. The slope decreases with increasing field which is parallel to the $c$-axis. Inset: Contact configuration used in our measurements.

face of the sample and the voltage drops between contacts on the same (primary voltage $V_{p}$ ) and the opposite (secondary voltage $V_{s}$ ) faces was measured for temperature, total current, and magnetic field applied parallel to the c-axis of the crystal in the ranges of $(1.9 \mathrm{~K} \leq T \leq 20 \mathrm{~K})$, $(0.3 \mu A \leq I \leq 2 m A)$, and $(0.2 T \leq H \leq 9 T)$. The single crystal, cleaved into a bar-shaped sample, was mounted on a single-crystal $\mathrm{MgO}$ substrate. $(\mathrm{MgO}$ and $\mathrm{YBa}_{2} \mathrm{Cu}_{3} \mathrm{O}_{7-\delta}$ have similar coefficients of thermal expansion.) The eight electrodes were fabricated by bonding 2 mil Au wire to the sample with Ag paste. A typical contact resistance was $2 \Omega$ or less. The mean-field superconducting transition temperature $T_{c 0} \simeq 16.9 \mathrm{~K}$ was determined from the temperature dependence of the inplane electrical resistance $R(T)$ measured in a magnetically shielded environment $\left(H<10^{-2} G\right)$ with a low transport current density $\left(<10 \mathrm{~A} / \mathrm{cm}^{2}\right)$ by using the $2 \mathrm{D}$ Coulomb gas model 25. At $T_{c 0}(\simeq 16.9 \mathrm{~K})$ the resistance of the sample is $90 \%$ that of the normal state value.

\section{A. Thermally assisted creep}

Figure 1 gives an overall view of the temperature and field dependence of the primary $V_{p}$ and secondary $V_{s}$ voltages. These voltages are normalized to the current and field. The convergence of these curves at $T \approx 9 \mathrm{~K}$ indicates a regime where the dissipation is due to the free motion of vortices. At lower temperatures, both resistances exhibit activated $T$-dependence with field-dependent activation energies. Due to the resistive anisotropy of the crystal, greater current flows near the primary face so that $V_{p}>V_{s}$ in both the normal and mixed states. At even lower $T$, the primary voltage becomes $T$ indepen- dent indicating onset of quantum creep while the secondary voltage remains thermally activated.

At $T \approx 9 K$, the resistance determined from both the primary and secondary voltages is proportional to the applied field, i.e.,

$$
R_{i} \propto R_{i}^{n} \frac{H}{H_{c 2}}
$$

where $R_{i} \equiv R_{p, s} \equiv V_{p, s} / I$ and $R_{i}^{n}$ is the corresponding normal state resistance. Equation (11) describes the free flow of vortices near the upper critical field $H_{c 2}(T)$ [19]. Fields of $0.2 T$ and higher are large enough, for this sample, to shift the onset of free flux flow regime substantially below the zero field $T_{c} \approx 17 \mathrm{~K}$.

The system of vortices undergoes a transition into a new state at a sharply defined temperature $T^{*}(H)$ which is the same for both primary and secondary voltages and decreases with increasing $H$ (Figs. 2(a) and 2(b)). For $T>T^{*}$, the activation energies near the primary and secondary faces of the crystal $\left(E_{p, s} \equiv-d \ln V_{p, s} / d(1 / T)\right)$ are equal and, therefore, current independent. Below $T^{*}$, both $E_{p}$ and $E_{s}$ change [both $V_{p, s}(T)$ curves acquire a different slope], but always $E_{s}>E_{p}$. The value of $T^{*}$ decreases strongly with increasing field.

It is interesting that the ratio $V_{p} / V_{s}$ which changes with temperature and field appears to scale with the values of $T^{*}$, as demonstrated in Inset in Fig. 2(a). Plotted versus $T / T^{*}$, the data points for different fields form a single curve. Note also that the transition to Tindependent creep takes place at $T=T_{q} \approx 0.55-0.6 T^{*}$. Such a strong correlation between $T_{q}$ and $T^{*}$ is a clear indication that the changes in the vortex system which occur at $T^{*}$ have a strong impact on the transition to quantum creep, or, perhaps, are a prerequisite for such a transition.

Further details of the transformation at $T^{*}$ are presented in Fig. 3, where the primary resistance $R_{p}(T)$ is shown for several values of the transport current within a range of over two decades $(1 \mu A \leq I \leq 250 \mu A)$. The dissipation at $T>T^{*}$ is ohmic, so that the resistance $R_{p}(T)$ and activation energy $E_{p}$ are current independent. However, the dissipation becomes non-ohmic below $T^{*}$. At low currents, the activation energy below $T^{*}$ is greater than above $T^{*}$, so that the curve $R_{p}(1 / T)$ has downward curvature. The activation energy decreases with increasing current, and, at sufficiently large currents, $E_{p}(I)$ becomes smaller than it is at $T>T^{*}$. For large enough currents, therefore, $R_{p}(1 / T)$ acquires upward curvature. This explains the upward curvature around $T^{*}$ of $R_{p}(T)$ in Fig. 2(a) (large current) and its downward curvature in Fig. 2(b) (smaller current). The secondary voltage $V_{s}$ reflects the substantially smaller current density reaching the secondary (bottom) surface of the sample. It shows some degree of non-ohmicity, but not as pronounced as the primary voltage. 


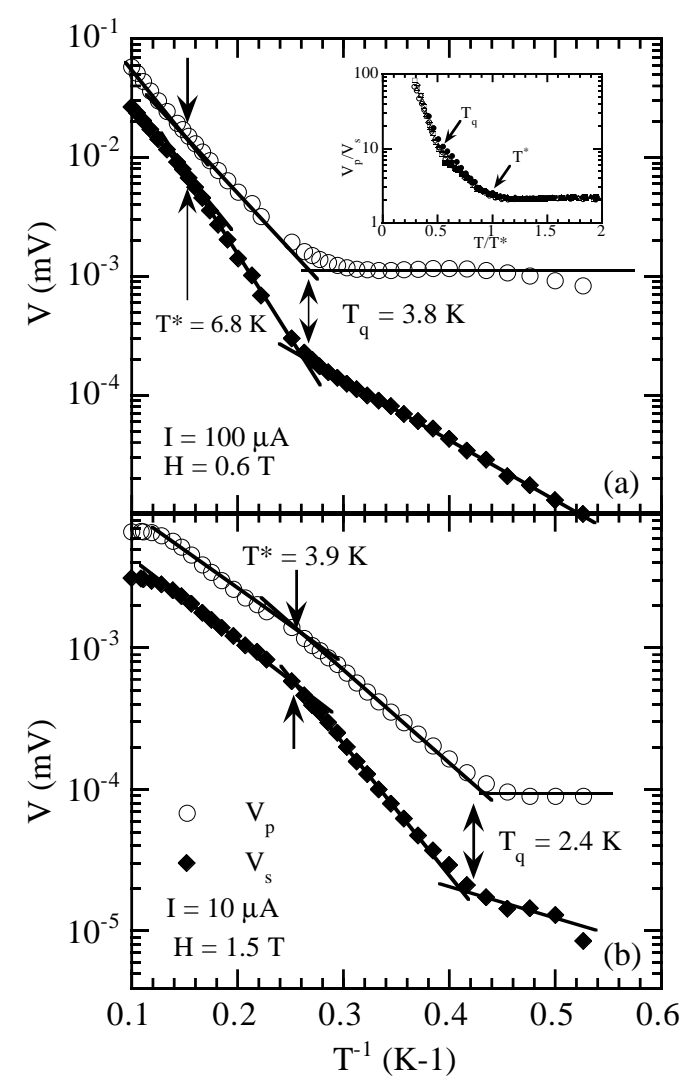

FIG. 2. Arrhenius plots of the primary $V_{p}$ and secondary $V_{s}$ voltages measured in two different fields and currents; (a) $0.6 \mathrm{~T}$ and $I=100 \mu \mathrm{A}$, and (b) $1.5 \mathrm{~T}$ and $I=10 \mu \mathrm{A}$. Inset to (a): The "anisotropy" $V_{p} / V_{s}$ plotted versus reduced temperature $T / T^{*}$ for applied magnetic fields $H$ of $0.2,0.4$, $0.6,0.8,1.0,1.5,2.0,2.5$, and $4.0 T$.

The kink at $T^{*}$ in $R_{p}$ (Figs. 2(a), 2(b) and 3) is similar to that observed in four-point resistive measurements on $\mathrm{Mo}_{77} \mathrm{Ge}_{33} / \mathrm{Ge}$ and $\mathrm{Mo} / \mathrm{Si}$ multilayers 2,3] and oxygen deficient $\mathrm{YBa}_{2} \mathrm{Cu}_{3} \mathrm{O}_{7-\delta}$ thin films [26]. However, the $\mathrm{Mo}_{77} \mathrm{Ge}_{33} / \mathrm{Ge}$ multilayers exhibit a downward curvature in $R(T)$ [2], while the $\mathrm{YBa}_{2} \mathrm{Cu}_{3} \mathrm{O}_{7-\delta}$ films show an upward curvature [26]; the $\mathrm{Mo} / \mathrm{Si}$ shows downward curvature for some samples and upward curvature for others [3]. The data in the Inset in Fig. 3 demonstrate that the origin of this contradiction is the current dependence of the activation energy below $T^{*}$. The threshold current at which the curvature of $R(T)$ changes sign is material and sample specific, which explains seemingly contradictory, in this respect, outcomes of different experiments.

Furthermore, Fig. 3 shows that the crossover temperature $T^{*}$ is current independent. This, along with the fact that $T^{*}$ is the same for both the primary and secondary voltages indicates a thermodynamic transition at $T^{*}$ rather than a kinetic phenomenon. All these results indicate that this thermodynamic transition is between a system of decoupled $2 \mathrm{D}$ vortices and a system of $3 \mathrm{D}$ vortices with the macroscopic coherence length along the direction of the magnetic field (the c-axis in this case).

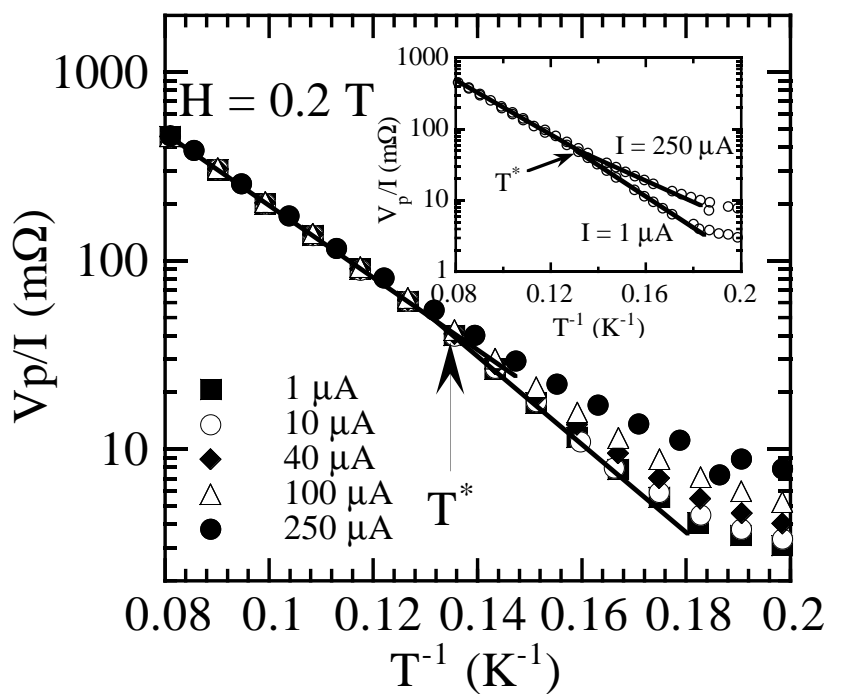

FIG. 3. Temperature dependence of the primary resistance $V_{p} / I$ for different values of the total current measured in a magnetic field $H=0.2 \mathrm{~T}$. The resistance is ohmic above $T^{*}$ and non-ohmic below $T^{*}$. For clarity, the inset shows the same data for two values of current, $1 \mu A$ and $250 \mu A$. The solid lines are guides to the eye.

At $T>T^{*}$, the vortices are not coherent in the direction of the magnetic field (decoupled) and behave as 2D "pancakes". The dissipation mechanism is activated hopping of $2 \mathrm{D}$ pancakes over potential barriers since the activation energies are the same for the primary and secondary surfaces in spite of the nonuniform current distribution. Figure 1 is a plot of the activation energy $U^{2 D} \equiv E_{p, s}$ versus field at $T>T^{*}$. $U^{2 D}(H)$ decreases monotonically with increasing field. This is expected because some of the vortices fill the deepest pinning wells. This leads, due to mutual repulsion, to a smoother potential profile (healing of the random potential) and, on average, lower activation energies for the rest of the vortices, which provide the bulk of the dissipation. The decrease of the activation energy due to healing is most pronounced at low vortex densities (low $H$ ) since the vortices heal the deepest parts of the pinning profile first. The efficiency of this process decreases at higher vortex densities (higher $\mathrm{H}$ ). Correspondingly, the rate of change, $d U^{2 D} / d H$, decreases with increasing field.

The pancake vortices form coherent lines at $T<T^{*}$ and, hence, the activation energy increases $\left(V_{p, s}\right.$ curve downward). However, the vortex lines do not extend through the whole thickness of the sample, so that $V_{p}$ remains greater than $V_{s}$ and the ratio $V_{p} / V_{s}$ even increases below $T^{*}$ (see Inset in Fig. 2(a)). Moreover, the activation energy decreases with increasing current and eventually becomes smaller than that for $2 \mathrm{D}$ pancake vortices ( $V_{p}$ acquires an upward curvature). This is clearly inconsistent with the idea that the correlation length $L_{c}$ is limited by "flux cutting" processes [27]. If $L_{c}$ along the c-axis is destroyed by the stress due to the driving 


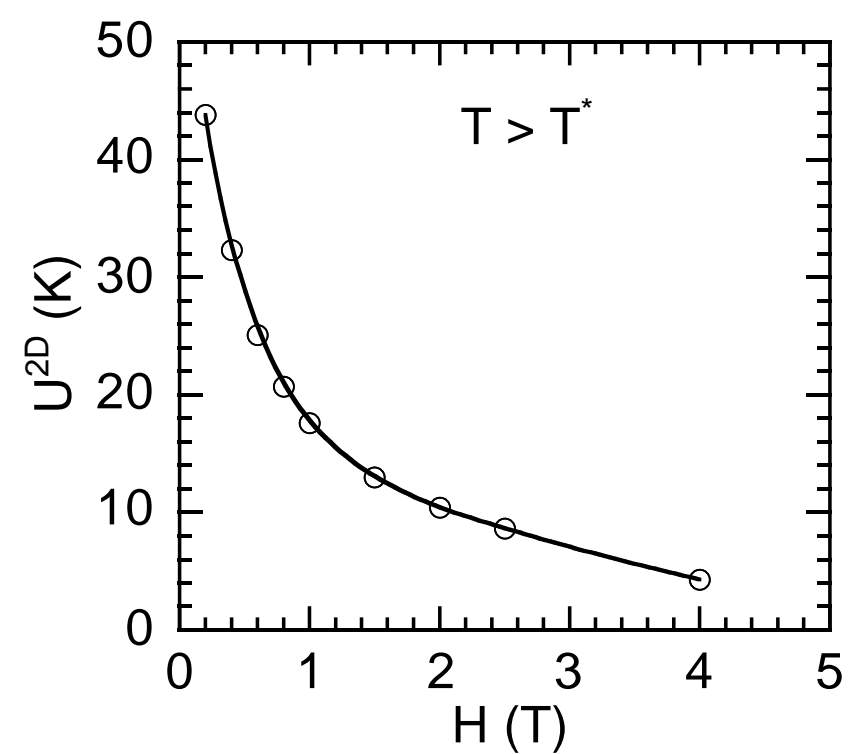

FIG. 4. Magnetic field dependence of the activation energy $U^{2 D} \equiv d \ln V_{p, s} / d(1 / T)$ determined at $T>T^{*}$. The solid line is a guide to the eye.

force, the activation energy would decrease with increasing current but could not become smaller than it is for $2 \mathrm{D}$ pancake vortices. It is obvious then, that below $T^{*}$ a new channel of relaxation opens up and becomes dominant at sufficiently large currents.

Both the field and current dependences of the activation energy at $T<T^{*}$ are consistent with a $3 D$ plastic creep model based on dislocation mediated motion of vortices, similar with diffusion of dislocations in atomic solids [28], with the activation energy given by [28,29]:

$$
U_{p l}(I, B)=U_{p l}^{o}(B)\left(1-\left(\frac{I}{I_{c}^{p l}}\right)^{m}\right),
$$

where $B$ is the magnetic induction, $U_{p l}^{o}(B)$ is the activation energy at $I=0$ and $I_{c}^{p l}$ is the critical current corresponding to the plastic motion of the vortices. In the limit of small currents, the activation energy $U_{p l}^{o}$ for the motion of a dislocation in a $3 \mathrm{D}$ vortex system can be estimated as the energy needed for the formation of a double kink over the Peierls barrier [22,29, 30]; i.e.,

$$
U_{p l}^{o}(B) \simeq \frac{2 a_{o} \epsilon_{o}}{\gamma}=\frac{\Phi_{o}^{2}}{8 \pi^{2} \gamma \lambda_{a b}^{2}}\left(\frac{\Phi_{o}}{B}\right)^{\frac{1}{2}},
$$

where $\epsilon_{o}=\left(\frac{\Phi_{o}}{4 \pi \lambda_{a b}}\right)^{2}$ is the line tension for a vortex aligned along the c-axis, $\Phi_{o}=2.07 \times 10^{-7} \mathrm{Gcm}^{2}$ is the fluxoid quantum, $a_{o}=\sqrt{\frac{\Phi_{o}}{B}}$ is the vortex lattice constant, $\gamma=\sqrt{\frac{\lambda_{c}}{\lambda_{a b}}}$ is the anisotropy parameter, and $\lambda_{a b, c}$ is the penetration depth associated with screening currents flowing in the ab plane and c-axis, respectively.

Figure $5\left(\right.$ a) is a log-log plot of $U_{s}^{3 D}$ versus $H$, extracted from the secondary voltage $V_{s}(T, H)$ where the current
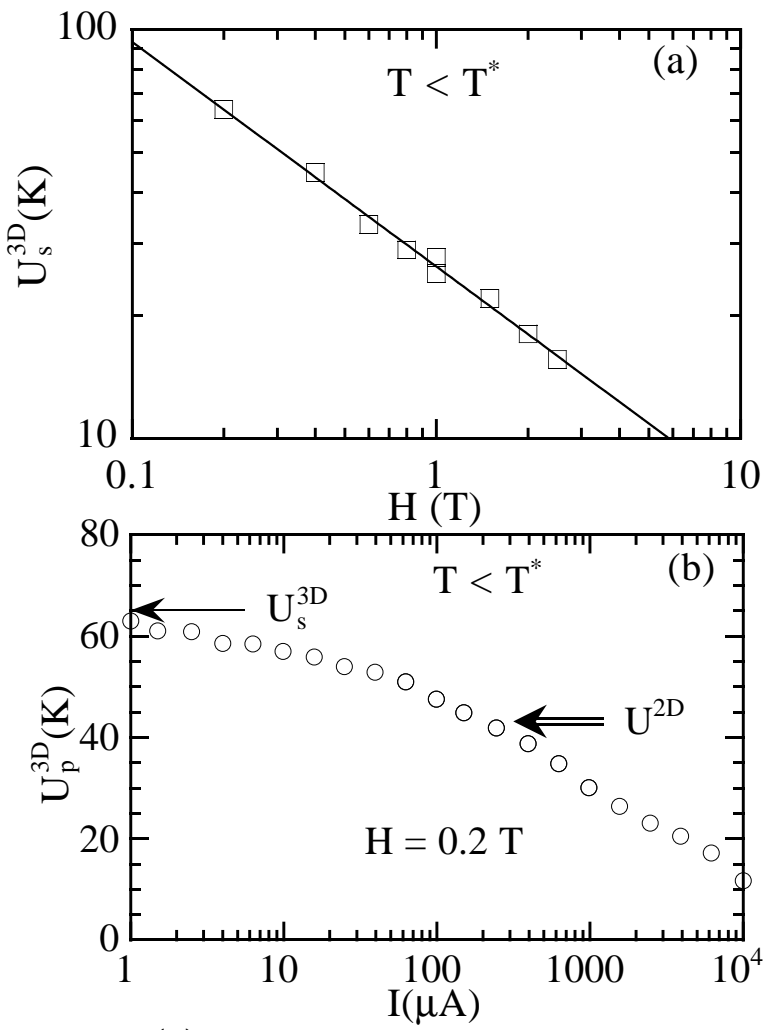

FIG. 5. (a) Field $H$ dependence of the activation energy $U^{3 D}$ determined from the secondary voltage as $d \ln V_{s} / d(1 / T)$ for $T<T^{*}$. The solid line is a fit of the data to Eq. (3).(b) Current $I$ dependence of the activation energy $U_{p}^{3 D}$ determined from the primary voltage as $d \ln V_{p} / d(1 / T)$ for $T<T^{*}$ in a field $H=0.2 T$. In the limit of small current, the value of $U_{p}^{3 D}$ is equal to that of $U_{s}^{3 D}$ determined from the secondary voltage in the same range of temperature $T<T^{*}$. The value of $U^{2 D}$ is also indicated by the double arrow.

is very small $(I \rightarrow 0)$. The data exhibit an $H^{-1 / 2}$ dependence which is characteristic of the motion of a dislocation in a 3D vortex structure (Eq. (30)). Figure 5(b) displays the current dependence of the activation energy $U_{p}^{3 D}=E_{p}$ extracted from the primary voltage $V_{p}(T, I)$ for $T<T^{*}$, measured in a magnetic field $H=0.2 T$. The double arrow indicates the value of the current indepen$\operatorname{dent} U^{2 D}$ at $T>T^{*}$ for the same magnetic field. Notice that $U^{3 D}>U^{2 D}$ for $I<0.1 m A$ and $U^{3 D}<U^{2 D}$ for $I>0.1 \mathrm{~mA}$. At this threshold current, $V_{p}(T)$ changes its curvature from downward open to upward open. In summary, these results show that the dissipation at $T<T^{*}$ is determined by two parallel processes: thermally activated motion of correlated vortices (dominant at low currents) with the activation energy greater than that for a $2 \mathrm{D}$ vortex, and plastic motion of dislocations (dominant at higher currents) with the activation energy smaller than that for a $2 \mathrm{D}$ vortex.

A schematic model of the flux flow which transpires from these observations is shown in Fig. 6. The current applied through the contacts on the primary face creates a nonuniform Lorentz force acting on the vortices, 


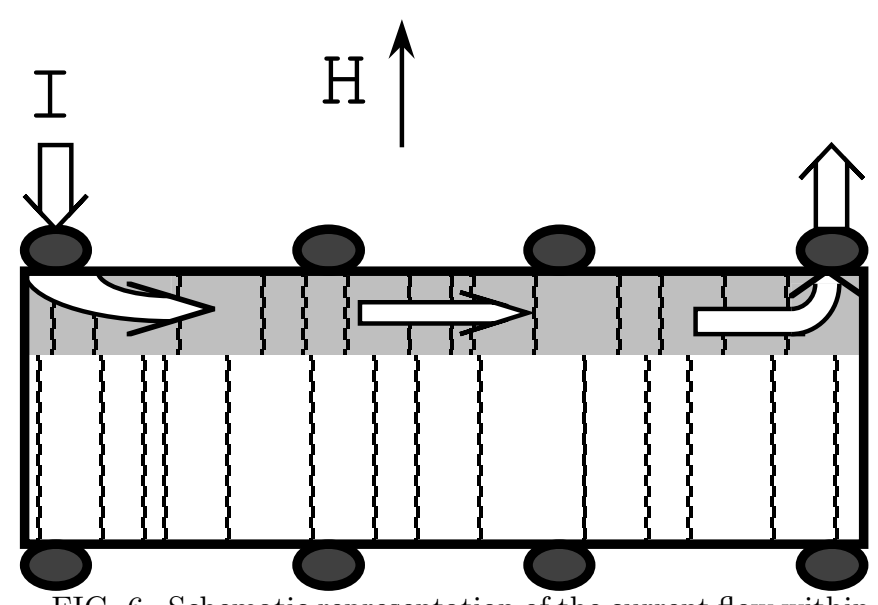

FIG. 6. Schematic representation of the current flow within the single crystal. The transport current flows mainly in the upper (shaded) layer. The vortices are coupled within each layer, but the two layers, current-carrying and "dormant" (unshaded) are decoupled from each other. The correlation length of vortices in the dormant layer is greater than that in the current-carrying layer.

which is equivalent to the application of a shear stress to a fragile solid at $T<T^{*}$. The shear stress triggers plastic flow which is strongest near the primary surface where the current density is greatest. On the other hand, the vortex lines near the secondary face remains relatively undisturbed. This difference of shear stress results in asymetric flux growth such that the vortices that grow from the secondary surface are longer than those that originate on the primary face. These two regions of the sample are uncoupled, yet for each of them the vortices are coherent over macroscopic distances. In this scenario, the large resistive anisotropy measured in the mixed state results from the loss of the phase coherence only between two macroscopic regions of the sample, not between all microscopic layers (such as $\mathrm{CuO}_{2}$ bilayers) as in the 2D phase at $T>T^{*}$.

\section{B. Quantum creep}

At lower temperatures, another transition at a field dependent temperature $T_{q}$ takes place (see Figs. 1, 2(a), and $2(\mathrm{~b}))$. The primary voltage $V_{p}$ becomes temperature independent and scales with the applied magnetic field; i.e., the resistance curves $R_{p}=V_{p} / I$ normalized to the magnetic field $R_{p} / H$ tend to converge below $T_{q}$ (Fig. 11). It is important to note that, although the secondary voltage (which represents the dissipation in the lower section of the sample) does not exhibit the transition to T-independent resistance, the activation energy is noticeably smaller below $T_{q}$ (Figs. 2(a) and 2(b)).

Since most of the current flows in a thin layer near the primary surface, it is useful to give an estimate of the value of the sheet resistance and residual mobility:

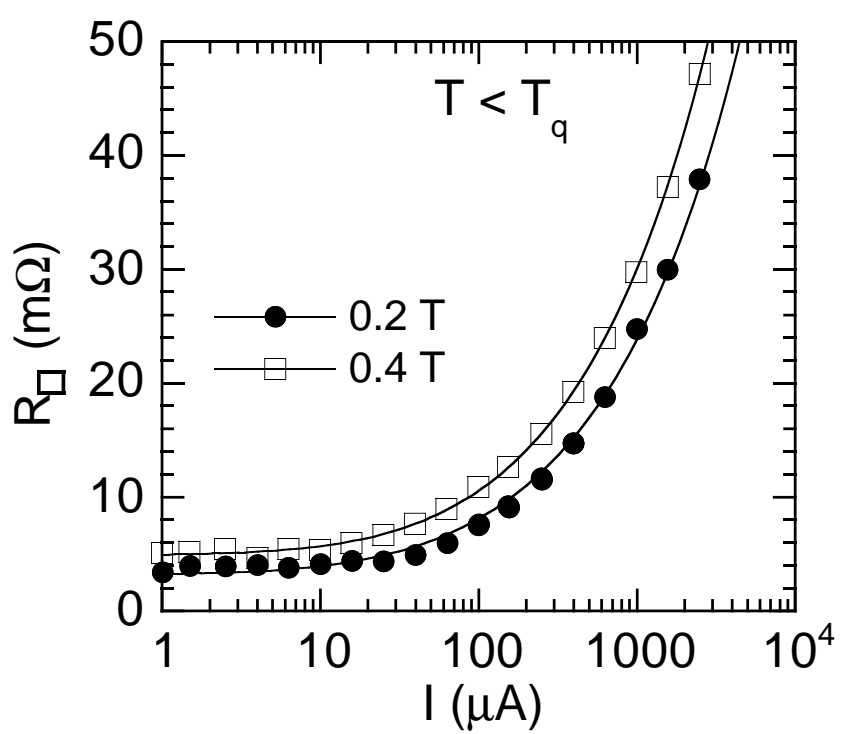

FIG. 7. Current $I$ dependence of the sheet resistance $R_{\square}$ in the quantum creep regime for two values of magnetic field 0.2 and $0.4 T$.

$$
R_{\square}=\frac{R_{p} b}{\ell} .
$$

Here $b \approx 0.5 \mathrm{~mm}$ is the width of the sample and $\ell \approx 0.3 \mathrm{~mm}$ is the distance between the voltage contacts. Following the conventional treatment 31] of the dissipation due to vortex drift, the sheet resistance can be expressed in terms of the vortex mobility $\mu_{\text {res }}$ (the total mobility of a moving segment, not the mobility per unit length):

$$
\mu_{\text {res }}=\frac{R_{\square} c^{2}}{\phi_{0} H},
$$

where $c$ is the speed of light and $\phi_{0}$ is the flux quantum. For $R_{\square} \propto H$ (Fig. 1), the vortices below $T_{q}$ are characterized by a finite, field independent "residual mobility" even in the limit $T \rightarrow 0$. The saturation of $V_{p}$ at low temperatures persists even at the lowest current of $0.01 \mathrm{~mA}$. At low currents, $R_{\square}=\mathcal{R} H$ with $\mathcal{R} \approx 20 \mathrm{~m} \Omega / T=6 \times 10^{-6} \hbar / \mathrm{e}^{2} T^{-1}$; the corresponding "residual mobility" $\mu_{\text {res }} \approx 1 \times 10^{10} \mathrm{~s} / \mathrm{g}$.

As shown in Fig. 目, the resistance $R_{\square}$ is current independent at lower currents and increases with increasing current at $I>10 \mu \mathrm{A}$. The current dependence of $R_{\square}$ can be well fitted with

$$
R_{\square}=H \mathcal{R}\left(1+\frac{I}{I_{0}}\right)^{1 / 2},
$$

with $I_{0} \approx 20 \mu \mathrm{A}$.

These observations clearly indicate that, in strongly underdoped $Y_{1-x} \mathrm{Pr}_{x} \mathrm{Ba}_{2} \mathrm{Cu}_{3} \mathrm{O}_{7-\delta}$, quantum creep begins to dominate classical, thermally activated creep at relatively high temperatures $T_{q} \sim 5 K$ in $H=0.2 T$. A factor that may facilitate a transition from classical to 
quantum creep is the high normal state resistivity. Previously, we measured the normal state resistivity $\rho_{n}(T)$ of the same sample by suppressing the superconductivity with a large magnetic field 18. This showed that the normal state of this superconductor is insulating, similar to that of $\operatorname{PrBa} a_{2} C u_{3} O_{7-\delta}$, so that $\rho_{n}(T) \rightarrow \infty$ as $T \rightarrow 0$. The reduced dissipation in the normal core of a vortex due to a large normal-state resistivity increases the mobility of the vortices [19] and, therefore, facilitates tunneling 20].

However, current theories cannot be directly applied to this system, because they predict the Euclidian action $S_{E}$ of the tunneling process to scale to zero with the zero temperature normal-state conductivity 32]; i.e.,

$$
\frac{S_{E}}{\hbar} \approx \frac{\hbar L_{c}}{e^{2} \rho_{n}(0)},
$$

where $L_{c}$ is the length of the tunneling segment. It is clear, however, from the small values of the sheet resistance and residual mobility, $R_{\square} \propto \mu_{\text {res }} \propto \exp \left\{-S_{E} / \hbar\right\}$, that the Euclidian action does not tend to zero, but remains finite at $T \rightarrow 0$. Hence, the theory of vortex tunneling, as well as the Bardeen-Stephen treatment of viscosity must be modified for systems which have insulating normal state underlying the superconductivity. A serious discrepancy between experimental and theoretical values of viscosity was also noted in Ref. [13] by the analysis of the relaxation rate in dirty superconductors.

Previously, the quantum creep in transport measurements was observed exclusively in thin films whose thickness did not exceed $30-40 \AA$ [14 16$]$. The main reason for this is the exponential decrease of the probability of tunneling with increasing correlation length along the field direction. In films, this length is restricted by the thickness of the film. Our crystals have a much greater thickness, about $1.5 \times 10^{5} \AA$. However, the fact that we observe a T-independent primary voltage $V_{p}$ and a thermally activated $V_{s}$ indicates that the thickness of the "upper" (current-carrying) layer (see Fig. 6) is probably self-restricted to just a few unit cells, thus facilitating tunneling of such short segments even at relatively high temperatures $(T \approx 5 K)$. The correlation length is much longer in the rest of the sample (below the current-carrying layer), comparable to the total thickness of the sample and, as a result, the vortices do not tunnel and the secondary voltage remains thermally activated (Figs. 11 and 2). It is interesting, however, that the transition to quantum creep still has an effect on the bulk of the crystal, because the activation energy determined by the slope of the secondary resistance $\left|d \ln R_{s} / d(1 / T)\right|$ decreases at $T_{q}$.

\section{RELAXATION OF MAGNETIZATION}

To verify that the transition to a temperature independent creep, discussed above, is due to quantum tunneling, we also performed magnetic relaxation measurements on another single crystal of $Y_{.47} \mathrm{Pr}_{.53} \mathrm{Ba}_{2} \mathrm{Cu}_{3} \mathrm{O}_{7-\delta}$, using a $S Q U I D$ magnetometer over a temperature range $2 K \leq T \leq 20 K$ for applied magnetic fields $H$ up to $5 \mathrm{~T}$. A small, $3 \mathrm{~cm}$, scanning length was used to minimize the variations in field strength inside the sample due to spatial inhomogeneities in the magnet $(\delta H<0.048 \%)$. The superconducting transition temperature $T_{c} \simeq 21 \mathrm{~K}$ of this single crystal was determined from the onset of diamagnetism measured in a low magnetic field $(H=10 \mathrm{Oe})$. The irreversibility temperature $T_{i r r}$ for a given $H$ was defined as the temperature above which the zero-field-cooled and field-cooled magnetic moments are identical. Magnetic relaxation measurements were performed by cooling the sample in zero field, applying a field $H+\Delta H(\Delta H=0.3 T$ for all $H)$ parallel to the c-axis of the crystal and then reducing it to $H$. The decay of the resultant paramagnetic moment was monitored for several hours $\left(\approx 10^{4} \mathrm{~s}\right)$ in constant field $H$. This procedure was used to ensure that the sample was in the fully critical state [33]. The irreversible part of the magnetic moment $M_{i r r}$ was obtained approximately by subtracting the field-cooled moment from the total measured moment.

From this data we can determine whether the relaxation process also exhibits a transition from thermally activated to quantum relaxation at the same temperature as the transport resistance. When the relaxation of the magnetic moment proceeds as a sequence of uncorrelated microscopic steps, each requiring thermal activation over an energy barrier, the decay time $\tau_{d}$ during which the induced moment loses a substantial fraction of its initial value can be expressed as:

$$
\tau_{d}=\tau_{e s c} \exp \left\{\frac{U(H, T)}{T}\right\} .
$$

Here the Boltzman factor reflects the degree of availability of energy $U$ required for an average elementary step to proceed and is essentially independent of the physics of the relaxation process. The pre-exponential factor $\tau_{e s c}$ is a measure of how rapidly the relaxation would proceed, had it not been limited by the unavailability of thermal energy. We call $\tau_{\text {esc }}$ an escape time to distinguish it from the microscopic attempt time $\tau_{a}$ which characterizes the period of vibration of the vortex inside a pinning well. The escape time depends on the size of the sample and may depend as well on the magnetic field and temperature. Factorization of the decay time given by Eq. (8) is meaningful as long as the Boltzman factor $\exp (U / T) \gg 1$, so that it dominates the temperature and field dependence of $\tau_{d}$. Since the activation energy van- 
ishes near $T_{c}$, the definition of $\tau_{d}$ can be specified further by taking a linear $T$-dependence of the effective barrier:

$$
U(H, T) \approx U_{0}(H)\left(1-\frac{T}{T_{c r}}\right)
$$

where $T_{c r}$ is the temperature at which the effective activation energy vanishes. It is commonly taken to be equal to the critical temperature, but may be smaller than $T_{c}$ and close to the irreversibility temperature. Thus, the decay time has the form:

$$
\tau_{d}=\tau_{e s c} \exp \left\{U_{0}(H)\left(\frac{1}{T}-\frac{1}{T_{c r}}\right)\right\} .
$$

We want to emphasize that Eq. (8) is more general than any particular dynamic model of the relaxation process driven by fluctuations. Therefore, it can also be obtained within the commonly used model in which the relaxation is described as a decay of the average supercurrent $J$ determined by current-dependent activation en$\operatorname{ergy} U(J, B, T)$ :

$$
\frac{d J}{d t}=-K \exp \left\{-\frac{U(J, H, T)}{T}\right\} .
$$

Since $U(J)$ increases with decreasing current, this equation can be integrated by the method of steepest descent:

$$
K t=\int_{J}^{J_{c}} d J^{\prime} \exp \left\{\frac{U\left(J^{\prime}\right)}{T}\right\} \approx \frac{T}{|d U / d J|} \exp \left\{\frac{U(J)}{T}\right\} .
$$

All unknown parameters can be absorbed into one $\tau_{\text {esc }}$ so that an approximate solution of Eq. (11) has the form

$$
\frac{U(J, H, T)}{T}=\ln \left(\frac{t}{\tau_{e s c}}\right)
$$

from which Eq. (8) immediately follows. Equation (13) was obtained earlier 34] by a more circuitous derivation.

With $U(J)$ given by the collective creep model [22],

$$
U(J, H, T)=\frac{U(H, T)}{\nu}\left(\left(\frac{J_{c}}{J}\right)^{\nu}-1\right),
$$

Eq. (13) gives the following time dependence of $J$ :

$$
J(t)=J_{c}\left(1+\nu \frac{T}{U} \ln \left(t / \tau_{\text {esc }}\right)\right)^{-1 / \nu} .
$$

On the other hand, for an arbitrary $U(J)$ in Eq. (13), the initial decay $\left(J_{c}-J \ll J_{c}\right)$ is linear in logarithm of time (Kim-Anderson formula):

$$
J(t)=J_{c}\left(1-\left(\frac{T}{U}\right) \ln \frac{t}{\tau_{e s c}}\right),
$$

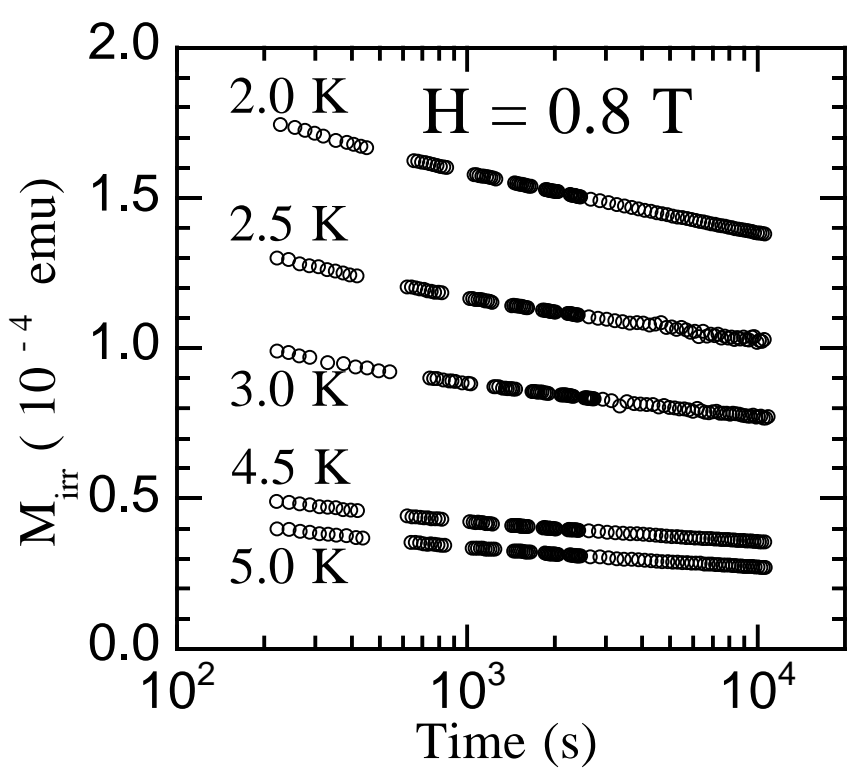

FIG. 8. Time $t$ dependence of the relaxation of the irreversible magnetic moment $M_{i r r}$ measured at different temperatures in a magnetic field $H=0.8 T$. To avoid clutter, only a few representative curves are shown.

where $U=J_{c}\left|d U / d J_{c}\right|$, the critical current is determined by the condition $U\left(J_{c}\right)=0$, and it is assumed that $d U / d J=$ const. at $J=J_{c}$.

Due to the slowness of relaxation, the decay time $\tau_{d}$ cannot be directly determined by monitoring the relaxation of the induced moment until it loses a substantial fraction of the initial value. An alternative method is to estimate the decay time by extrapolating the initial decay of $J(t)$ to lower current values. Specifically, when the initial decay is described by Eq. (16), we define $\tau_{d}$ from the condition $J\left(\tau_{d}\right)=0$. Comparing this definition with the collective creep formula, Eq. (15), we see that the so defined $\tau_{d}$ corresponds to a decay to the level of $J_{c} /(1+\nu)^{1 / \nu}$. The current density $J$ can be experimentally determined by the irreversible part of the magnetic moment $M_{i r r}(t) \propto J(t)$.

Representative semilog plots of $M_{i r r}(t) \propto J(t)$ as a function of time $t$ for several temperatures measured in a field $H=0.8 T$ are shown in Fig. 8. Within a decade of time $10^{3}-10^{4} \mathrm{~s}$, the relaxation curves can be well fitted to:

$$
M_{i r r}=a-b \ln \left(t / t_{0}\right)
$$

where $t_{0}$ is an arbitrary unit of time. The decay time for which $M_{i r r}\left(\tau_{d}\right)=0$ is then given by:

$$
\tau_{d}=t_{0} \exp \left\{\frac{a}{b}\right\} .
$$

With this definition, $\tau_{d}$ is universal and does not depend on the choice of $t_{0}$.

Figure 9 shows the decay time calculated according to Eq. (18) and plotted against the inverse temperature for 


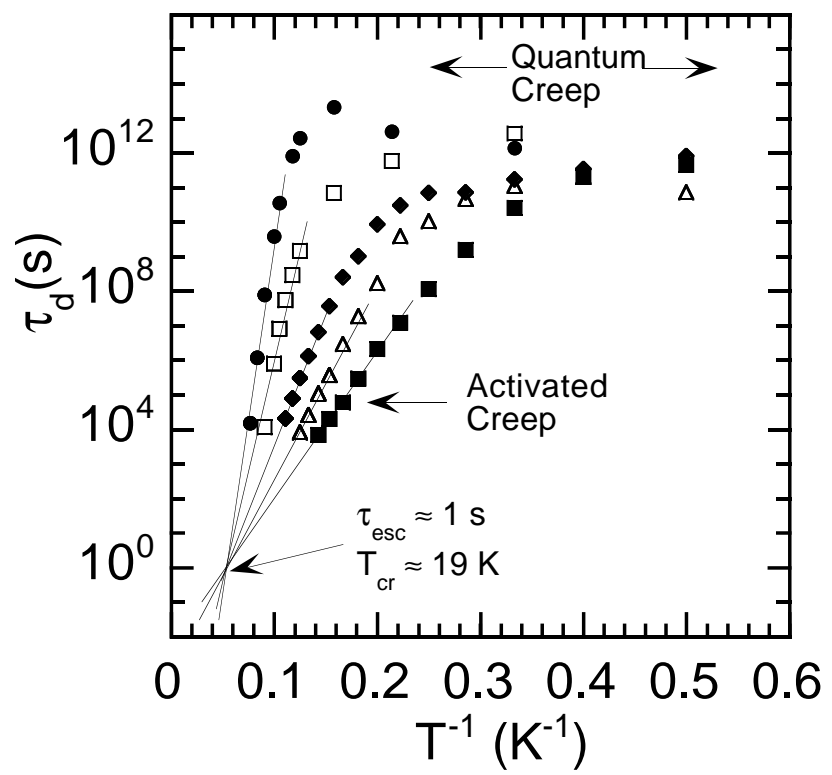

FIG. 9. Decay time $\tau_{d}$ versus $1 / T$ for several values of magnetic field $(H=0.1,0.2,0.6,0.8$, and $1.2 T)$. The slope decreases with increasing field. The straight line extrapolations of the Arrhenius type dependence converge at $T_{c r} \approx 19 \mathrm{~K}$ and $\tau_{d}=\tau_{\text {esc }} \approx 1 \mathrm{~s}$. The saturation of $\tau_{d}$ at the level $10^{11}-10^{12} \mathrm{~s}$ is due to quantum creep.

different values of magnetic field. A comparison of these data with $\tau_{d}(T)$ given by Eq. (10) allows us to determine whether the relaxation crosses over from activated to non-activated dependence, and at what temperature. At higher temperatures, indeed, the data display an Arrhenius dependence with a slope $d \ln \tau_{d} / d(1 / T)$ decreasing with increasing field. This trend is consistent with the field dependence of the activation energy in transport measurements, Fig. 且.

It is important to note that the values of the activation energy determined by the slopes $d \ln \tau_{d} / d(1 / T)$ are not universal and depend on the criterion used to define the decay time (see Eq. (13)). However, the pre-exponential factor $\tau_{e s c}$ is universal and can be determined by the extrapolation of the Arrhenius dependence of $\tau_{d}(T)$ to the temperature $T_{c r}$. Indeed, a linear extrapolation of the data in the activated region to higher temperatures (straight lines in Fig. 9) shows that the lines converge at $T_{c r} \approx 19 \mathrm{~K}$ (which is consistent with the value of $T_{c}$ or $\left.T_{i r r}\right)$. The point of convergence corresponds to $\tau_{d}=\tau_{e s c} \approx 1 \mathrm{~s}$. This is an extremely large characteristic time of relaxation in comparison with the attempt time which is typically assumed to be of the order of $10^{-9}-10^{-12} \mathrm{~s}$. The value of the escape time can be estimated from the following consideration. The decay of the induced moment occurs when vortices leave the sample [35]. Over long times (in comparison with the microscopic time scale) any type of rearrangement of vortices reduces to diffusion. Correspondingly, the escape time can be estimated as the time required for a vortex to diffuse from the bulk to the outer edge of the sample:

$$
\tau_{e s c} \sim \frac{R^{2}}{\mathcal{D}_{v}} \sim \frac{R^{2}}{\omega_{a} \ell_{a}^{2}} \equiv \frac{R^{2} m^{*}}{\hbar},
$$

where $R$ is the characteristic size of the sample in the direction of diffusion (in the $a-b$ plane in our case), and $\mathcal{D}_{v}$ is the diffusion coefficient determined by the attempt frequency $\omega_{a}$ and the average elementary vortex hopping distance $\ell_{a}$. With $\tau_{e s c} \sim 1 s$ and $R^{2} \sim$ $10^{-2}-10^{-3} \mathrm{~cm}^{2}$ (for the crystal we measured), Eq. (19) gives $\mathcal{D}_{v} \sim 10^{-2}-10^{-3} \mathrm{~cm}^{2} / \mathrm{s}$. This value of $\mathcal{D}_{v}$ is consistent with an elementary step of the order of the correlation length $\ell_{a} \sim 100 \AA$ and $\omega_{a} \sim 10^{10}-10^{9} \mathrm{~s}^{-1}$. We define the effective mass $m^{*}$ of a segment of the vortex line through the uncertainty principle, $\omega_{a} \sim \hbar / m^{*} \ell_{a}^{2}$. With these estimates, the effective mass of the diffusing vortex segment is $10^{2}-10^{3}$ times of the electron mass.

\section{A. Quantum creep}

At lower temperatures, the decay time saturates at a roughly temperature and field-independent level (Fig. 9). The crossover temperatures $T_{q}(H)$ from transport (Fig. 1) and magnetic relaxation (Fig. 9) measurements are very close in spite of a very large difference in the currents involved in these measurements. The fact that the transition to a temperature independent dissipation takes place in both transport and magnetic relaxation processes, and at approximately the same temperature in a given field indicates that both phenomena have a common origin.

In the regime of quantum relaxation, the relaxation rate is limited by the probability of tunneling as determined by the Euclidian action $S_{E}$. Similar to Eq. (11), the relaxation rate of the supercurrent can be expressed in terms of the current dependent $S_{E}(J)$ :

$$
\frac{d J}{d t}=-K \exp \left\{-\frac{S_{E}(J, H)}{\hbar}\right\}
$$

which has a solution similar to Eq. (13):

$$
\frac{S_{E}(J)}{\hbar}=\ln \left(\frac{t}{\tau_{e s c}}\right) \text {. }
$$

Provided that $d S_{E} / d J=$ const at $J_{c}$, where $J_{c}$ is determined by the condition $S_{E}\left(J_{c}\right)=0$, the initial decay has same linear in logarithm of time dependence as in the classical case (see Eq. (16)); i.e.,

$$
J(t)=J_{c}\left(1-\frac{\hbar}{S_{0}} \ln \frac{t}{\tau_{e s c}}\right),
$$

where $S_{0} \equiv J_{c}\left|d S_{E} / d J_{c}\right|$. The decay time determined by the extrapolation of Eq. (22) to $J\left(\tau_{d}\right)=0$ is given by:

$$
\tau_{d}=\tau_{e s c} \exp \left\{\frac{S_{0}}{\hbar}\right\} .
$$


The value of the escape time should be similar to that in the classical regime since it is determined by the diffusion uninhibited neither by the lack of the thermal energy, nor by the small tunneling probability. Therefore, we can estimate $S_{0}$ from the data of Fig. 9 :

$$
\frac{S_{0}}{\hbar}=\ln \left(\frac{\tau_{d}}{\tau_{\text {esc }}}\right) \approx 25 .
$$

This value is comparable, but somewhat smaller than those reported for other systems [13].

\section{SUMMARY AND SPECULATIONS}

We have observed quantum creep in underdoped $Y_{1-x} \mathrm{Pr}_{x} \mathrm{Ba}_{2} \mathrm{Cu}_{3} \mathrm{O}_{7-\delta}$ crystals using both transport and magnetic relaxation measurements. The transition to quantum creep is preceded by a coupling transition which leads to non-ohmic dissipation. The evidence presented in previous sections lead us to a picture of the current density distribution shown in Fig. 6. Most of the transport current is confined to a very thin layer below the current contacts. This current-carrying layer is decoupled from the rest of the crystal, where the vortices are mostly undisturbed by the current and are coherent over a macroscopically long distance, perhaps comparable to the thickness of this "dormant" layer which is practically the same as the thickness of the sample.

The transition from thermally activated to temperature independent dissipation takes place only in the current-carrying layer. The relatively large tunneling probability which makes possible the observation of this crossover at $T \approx 5 K$ in these crystals is due to very short tunneling segments, large normal state resistivity, and a large current density. In the rest of the sample, the vortices are much longer and their tunneling is suppressed as manifested by activated T-dependence of the secondary voltage down to the lowest temperature. This is consistent with the fact that quantum creep was previously observed only in transport experiments on ultrathin films and multilayers with a thickness no more than $30-40 \AA$.

Magnetic relaxation measurements substantiate that this T-independent resistance is due to quantum creep. The decay time of the magnetic moment becomes Tindependent at approximately the same temperatures (in a given magnetic field) as in transport (see Figs. 1, 2, and 9). We also determined the characteristic relaxation time $\tau_{e s c}$ which turns out to be very large $\sim 1 s$ in comparison with the microscopic attempt time.

While the finding of nonvanishing resistance at $T \rightarrow 0$ in a crystal and its correlation with nonvanishing magnetic relaxation rate is important and has never been observed before, our results raise also another important question. In the normal and mixed state above the temperature of the coupling transition $T^{*}$, the crystals of $\mathrm{Y}_{1-x} \mathrm{Pr}_{x} \mathrm{Ba}_{2} \mathrm{Cu}_{3} \mathrm{O}_{7-\delta}$ are not very anisotropic.
In the sample with the length $L \approx 1 \mathrm{~mm}$ and thickness $D \approx 0.015 \mathrm{~mm}$, the ratio $V_{p} / V_{s} \sim 2$, (see Inset in Fig. 2(a)) so that the transport current fills fairly uniformly the whole cross-section. On the other hand, the transition to quantum creep indicates that below $T_{q}$ the current-carrying volume collapses into a thin layer, possibly just a few unit cells thick. This favors the quantum creep for two reasons: minimum length of the vortex segments and maximum current density which reduces the height of pinning barriers. This opens the question of the nature of such a drastic self-channeling of the transport current.

A possible answer to this question is related to the nonohmic, current-dependent resistive anisotropy. This can be illustrated by the following qualitative dimensional considerations. According to local electrodynamics, in the sample with thickness $D$ (Inset in Fig. 1), the transport current mostly flows within a layer of thickness $D_{\text {eff }}$ 36:

$$
D_{e f f} \approx \frac{D}{\eta} ; \quad \eta \equiv \frac{\pi D}{L}\left(\frac{\rho_{c}}{\rho_{a b}}\right)^{1 / 2} ; \quad \eta>1 .
$$

If the effective anisotropy increases with increasing current density $j$ then the transport current exhibits a tendency to channel itself into an increasingly narrow layer as follows. Let us assume that to the lowest order in $j$, the anisotropy can be written as:

$$
\eta \approx \eta_{0}\left(1+\frac{j^{2}}{j_{0}^{2}}\right)
$$

where

$$
j=\frac{I}{D_{e f f}}
$$

and $I$ is the total current. Combining Eqs. (25) - (27), we obtain the following equation for the effective thickness of the current distribution:

$$
D_{e f f}=\frac{D}{\eta_{0}\left(1+\frac{I^{2}}{D_{e f f}^{2} j_{0}^{2}}\right)} .
$$

This equation has the solution

$$
D_{e f f}=\frac{D}{2 \eta_{0}}\left[1+\sqrt{1-\frac{I^{2}}{I_{\text {ins }}}}\right]
$$

where $I_{\text {ins }}=D j_{0} / 2 \eta_{0}$. As the total current $I$ increases, $D_{\text {eff }}(I)$ gradually decreases until it reaches half of its zero current value $D / \eta_{0}$ at $I=I_{\text {ins }}$. For $I>I_{\text {ins }}$, Eq. (28) does not have a real solution except for $D_{\text {eff }}=0$. Thus, $I_{\text {ins }}$ is a threshold of instability (the corresponding value of $\left.\eta_{\text {ins }}=\eta\left(I_{i n s}\right)=2 \eta_{0}\right)$. For $I>I_{\text {ins }}$ there is no stable current distribution with macroscopic thickness. The current-carrying layer compresses itself until it is a few 
unit cells thick, or until the current density approaches the critical value. The existense of such an instability would have significant implications to our understanding of the electrical transport in layered superconductors and their applications.

\section{ACKNOWLEDGMENTS}

This research was supported at KSU by the National Science Foundation under Grant Nos. DMR-9601839 and DMR-9801990, and at UCSD by U.S. Department of Energy under Grant No. DE-FG03-86ER-45230.

[1] I. Giaever, Phys. Rev. Lett. 15, 825 (1965).

[2] W. R. White, A Kapitulnik, and M. R. Beasley, Phys. Rev. Lett. 66, 2826 (1991).

[3] N. Y. Fogel, V. G. Cherkasova, O. A. Koretzkaya, and A. S. Sidorenko, Phys. Rev. B 55, 85 (1997).

[4] H. Safar, P. L. Gammel, D. A. Huse, S. N. Majumdar, L. F. Schneemeyer, D. J. Bishop, D. Lopez, G. Nieva, and F. de la Cruz, Phys. Rev. Lett. 72, 1272 (1994).

[5] R. Busch, G. Ries, H. Werthner, G. Kreiselmeyer, and G. Saemann-Ischecnko, Phys. Rev. Lett. 69, 522 (1992).

[6] H. Safar, E. Rodriguez, F. de la Cruz, P. L. Gammel, L. F. Schneemeyer, and D. J. Bishop, Phys. Rev. B 46, 14238 (1992).

[7] R. A. Doyle et al. Phys. Rev. Lett. 77, 1155 (1996).

[8] C. D. Keener et al. Phys. Rev. B 55, R708 (1997).

[9] Y. Eltsev, W. Holm, and O. Rapp, Phys. Rev. B 49, 12333 (1994).

[10] A. C. Mota et al. Progress in High Temperature Superconductivity, Proceedings of ICTPS 90, ed. by R. Nicolsky (World Scientific, Singapore, 1990), Vl. 25.

[11] A. C. Mota et al. Physica C, 185-189, 343 (1991) and references therein.

[12] A. J. J. van Dalen, R. Griessen, S. Libbrecht, Y. Bruynseraede, and E. Osquiguil, Phys. Rev. B 54, 1366 (1996); and references therein.

[13] A. F. Th. Hoekstra, R. Griessen. A. M. Testa, J. el Fattahi, M. Brinkmann, K. Westerholt, W. K. Kwok, and G. W. Crabtree, Phys. Rev. Lett. 80, 4293 (1998).

[14] Y. Liu, D. B. Haviland, L. I. Glazman, and A. M. Goldman, Phys. Rev. Lett. 68, 2224 (1992).

[15] D. Ephron, A. Yazdani, A. Kapitulnik, and M. R. Beasley, Phys. Rev. Lett. 76, 1529 (1996).

[16] J. A. Chervenak and J. M. Valles, Jr., Phys. Rev. B, 54, R15649 (1996).

[17] A. Gerber and J. M. Franse, Phys. Rev. Lett. 71, 1895 (1993).

[18] G. A. Levin, T. Stein, C. C. Almasan, S. H. Han, D. A. Gajewski, and M. B. Maple, Phys. Rev. Lett. 80, 841 (1998).
[19] J. Bardeen and M. J. Stephen, Phys. Rev. 140, 1197A (1965).

[20] A. O. Caldeira and A. J. Leggett, Ann. Phys. (N.Y.) 149, 374 (1983).

[21] G. Blatter, V. B. Geshkenbein, and V. M. Vinokur, Phys. Rev. Lett. 66, 3297 (1991).

[22] G. Blatter, M. V. Feigel'man, V. B. Geshkenbein, A. I. Larkin, and V. M. Vinokur, Rev. Mod. Phys. 66, 1125 (1994).

[23] T. Stein, G. A. Levin, C. C. Almasan, D. A. Gajewski, and M. B. Maple, Phys. Rev. Lett. 82, 2955 (1999).

[24] L. M. Paulius, B. W. Lee, M. B. Maple, and P. K. Tsai, Physica C 230, 255 (1994).

[25] P. Minnhagen and P. Olsson, Phys. Scripta T 42, 9 (1992).

[26] X. G. Qiu, B. Wuyts, M. Maenhoudt, V. V. Moshchalkov, and Y. Bruynseraede, Phys. Rev. B 52, 559 (1995).

[27] D. Lopez, G. Nieva, F. de la Cruz, H. J. Jensen, and D. O'Kane, Phys. Rev. B 50, 9684 (1994).

[28] J. P. Hirth and J. Lothe, Theory of Dislocations (John Wiley \& Sons, New York, 1982), Chap. 15.

[29] Y. Abulafia, A. Shaulov, Y. Wolfus, R. Prozorov, L. Burlachkov, Y. Yeshurun, D. Majer, E. Zeldov, H. Wuhl, V. B. Geshkenbein, and V. M. Vinokur, Phys. Rev. Lett. 77, 1596 (1996).

[30] V. B. Geshkenbein, A. I. Larkin, M. V. Feigel'man, and M. V. Vinokur, Physica C 162-164, 239 (1989).

[31] M. Tinkham, Introduction to Superconductivity (McGraw-Hill, Inc., 1996), p. 166.

[32] G. Blatter and V. B. Geshkenbein, Phys. Rev. B 47, 2725 (1993).

[33] For a review see Y. Yeshurun, A. P. Malozemoff, and A. Shaulov, Rev. Mod. Phys. 68, 911 (1996).

[34] V. B. Geshkenbein and A. I. Larkin, Zh. Eksp. Teor. Phys. 95,1108 (1989); Sov. Phys. JETP 68, 639 (1989).

[35] M. V. Feigel'man, V. B. Geshkenbein, and V. M. Vinokur, Phys. Rev. B 43, 6263 (1991).

[36] G. A. Levin, J. Appl. Phys. 81, 714 (1997) 